\title{
Data Mining Approaches for Intrusion Detection*
}

\author{
Wenke Lee Salvatore J. Stolfo \\ Computer Science Department \\ Columbia University \\ 500 West 120th Street, New York, NY 10027 \\ \{wenke,sal\}@cs.columbia.edu
}

\begin{abstract}
In this paper we discuss our research in developing general and systematic methods for intrusion detection. The key ideas are to use data mining techniques to discover consistent and useful patterns of system features that describe program and user behavior, and use the set of relevant system features to compute (inductively learned) classifiers that can recognize anomalies and known intrusions. Using experiments on the sendmail system call data and the network tcpdump data, we demonstrate that we can construct concise and accurate classifiers to detect anomalies. We provide an overview on two general data mining algorithms that we have implemented: the association rules algorithm and the frequent episodes algorithm. These algorithms can be used to compute the intra- and inter- audit record patterns, which are essential in describing program or user behavior. The discovered patterns can guide the audit data gathering process and facilitate feature selection. To meet the challenges of both efficient learning (mining) and real-time detection, we propose an agent-based architecture for intrusion detection systems where the learning agents continuously compute and provide the updated (detection) models to the detection agents.
\end{abstract}

\section{Introduction}

As network-based computer systems play increasingly vital roles in modern society, they have become the targets of our enemies and criminals. Therefore, we need to find the best ways possible to protect our systems.

The security of a computer system is compromised when

* This research is supported in part by grants from DARPA (F30602-96-1-0311) and NSF (IRI-96-32225 and CDA-96-25374) an intrusion takes place. An intrusion can be defined [HLMS90] as "any set of actions that attempt to compromise the integrity, confidentiality or availability of a resource". Intrusion prevention techniques, such as user authentication (e.g. using passwords or biometrics), avoiding programming errors, and information protection (e.g., encryption) have been used to protect computer systems as a first line of defense. Intrusion prevention alone is not sufficient because as systems become ever more complex, there are always exploitable weakness in the systems due to design and programming errors, or various "socially engineered" penetration techniques. For example, after it was first reported many years ago, exploitable "buffer overflow" still exists in some recent system software due to programming errors. The policies that balance convenience versus strict control of a system and information access also make it impossible for an operational system to be completely secure.

Intrusion detection is therefore needed as another wall to protect computer systems. The elements central to intrusion detection are: resources to be protected in a target system, i.e., user accounts, file systems, system kernels, etc; models that characterize the "normal" or "legitimate" behavior of these resources; techniques that compare the actual system activities with the established models, and identify those that are "abnormal" or "intrusive".

Many researchers have proposed and implemented different models which define different measures of system behavior, with an ad hoc presumption that normalcy and anomaly (or illegitimacy) will be accurately manifested in the chosen set of system features that are modeled and measured. Intrusion detection techniques can be categorized into misuse detection, which uses patterns of wellknown attacks or weak spots of the system to identify intrusions; and anomaly detection, which tries to determine whether deviation from the established normal us- 
age patterns can be flagged as intrusions.

Misuse detection systems, for example [KS95] and STAT [IKP95], encode and match the sequence of "signature actions" (e.g., change the ownership of a file) of known intrusion scenarios. The main shortcomings of such systems are: known intrusion patterns have to be hand-coded into the system; they are unable to detect any future (unknown) intrusions that have no matched patterns stored in the system.

Anomaly detection (sub)systems, such as

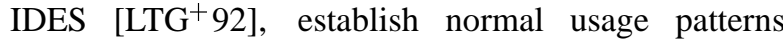
(profiles) using statistical measures on system features, for example, the CPU and I/O activities by a particular user or program. The main difficulties of these systems are: intuition and experience is relied upon in selecting the system features, which can vary greatly among different computing environments; some intrusions can only be detected by studying the sequential interrelation between events because each event alone may fit the profiles.

Our research aims to eliminate, as much as possible, the manual and ad-hoc elements from the process of building an intrusion detection system. We take a data-centric point of view and consider intrusion detection as a data analysis process. Anomaly detection is about finding the normal usage patterns from the audit data, whereas misuse detection is about encoding and matching the intrusion patterns using the audit data. The central theme of our approach is to apply data mining techniques to intrusion detection. Data mining generally refers to the process of (automatically) extracting models from large stores of data [FPSS96]. The recent rapid development in data mining has made available a wide variety of algorithms, drawn from the fields of statistics, pattern recognition, machine learning, and database. Several types of algorithms are particularly relevant to our research:

Classification: maps a data item into one of several predefined categories. These algorithms normally output "classifiers", for example, in the form of decision trees or rules. An ideal application in intrusion detection will be to gather sufficient "normal" and "abnormal" audit data for a user or a program, then apply a classification algorithm to learn a classifier that will determine (future) audit data as belonging to the normal class or the abnormal class;

Link analysis: determines relations between fields in the database. Finding out the correlations in audit data will provide insight for selecting the right set of system features for intrusion detection;

Sequence analysis: models sequential patterns. These algorithms can help us understand what (timebased) sequence of audit events are frequently encountered together. These frequent event patterns are important elements of the behavior profile of a user or program.

We are developing a systematic framework for designing, developing and evaluating intrusion detection systems. Specifically, the framework consists of a set of environment-independent guidelines and programs that can assist a system administrator or security officer to

- select appropriate system features from audit data to build models for intrusion detection;

- architect a hierarchical detector system from component detectors;

- update and deploy new detection systems as needed.

The key advantage of our approach is that it can automatically generate concise and accurate detection models from large amount of audit data. The methodology itself is general and mechanical, and therefore can be used to build intrusion detection systems for a wide variety of computing environments.

The rest of the paper is organized as follows: Section 2 describes our experiments in building classification models for sendmail and network traffic. Section 3 presents the association rules and frequent episodes algorithms that can be used to compute a set of patterns from audit data. Section 4 briefly highlights the architecture of our proposed intrusion detection system. Section 5 outlines our future research plans.

\section{Building Classification Models}

In this section we describe in detail our experiments in constructing classification models for anomaly detection. The first set of experiments, first reported in [LSC97], is on the sendmail system call data, and the second is on the network tcpdump data.

\subsection{Experiments on sendmail Data}

There have been a lot of attacks on computer systems that are carried out as exploitations of the design and 
programming errors in privileged programs, those that can run as root. For example, a flaw in the finger daemon allows the attacker to use "buffer overflow" to trick the program to execute his malicious code. Recent research efforts by Ko et al. [KFL94] and Forrest et al. [FHSL96] attempted to build intrusion detection systems that monitor the execution of privileged programs and detect the attacks on their vulnerabilities. Forrest et al. discovered that the short sequences of system calls made by a program during its normal executions are very consistent, yet different from the sequences of its abnormal (exploited) executions as well as the executions of other programs. Therefore a database containing these normal sequences can be used as the "self" definition of the normal behavior of a program, and as the basis to detect anomalies. Their findings motivated us to search for simple and accurate intrusion detection models.

Stephanie Forrest provided us with a set of traces of the sendmail program used in her experiments [FHSL96]. We applied machine learning techniques to produce classifiers that can distinguish the exploits from the normal runs.

\subsubsection{The sendmail System Call Traces}

The procedure of generating the sendmail traces were detailed in [FHSL96]. Briefly, each file of the trace data has two columns of integers, the first is the process ids and the second is the system call "numbers". These numbers are indices into a lookup table of system call names. For example, the number "5" represents system call open. The set of traces include:

Normal traces: a trace of the sendmail daemon and a concatenation of several invocations of the sendmail program;

Abnormal traces: 3 traces of the sscp (sunsendmailcp) attacks, 2 traces of the syslog-remote attacks, 2 traces of the syslog-local attacks, 2 traces of the decode attacks, 1 trace of the $s m 5 x$ attack and 1 trace of the $s m 565 a$ attack. These are the traces of (various kinds of) abnormal runs of the sendmail program.

\subsubsection{Learning to Classify System Call Sequences}

In order for a machine learning program to learn the classification models of the "normal" and "abnormal" system call sequences, we need to supply it with a set of

\begin{tabular}{|l|l|}
\hline System Call Sequences (length 7) & Class Labels \\
\hline 426666413866 & "normal" \\
\hline$\ldots$ & $\ldots$ \\
\hline 555459105104 & "abnormal" \\
\hline$\ldots$ & $\ldots$ \\
\hline
\end{tabular}

Table 1: Pre-labeled System Call Sequences of Length 7

training data containing pre-labeled "normal" and "abnormal" sequences. We use a sliding window to scan the normal traces and create a list of unique sequences of system calls. We call this list the "normal" list. Next, we scan each of the intrusion traces. For each sequence of system calls, we first look it up in the normal list. If an exact match can be found then the sequence is labeled as "normal". Otherwise it is labeled as "abnormal" (note that the data gathering process described in [FHSL96] ensured that the normal traces include nearly all possible "normal" short sequences of system calls, as new runs of sendmail failed to generate new sequences). Needless to say all sequences in the normal traces are labeled as "normal". See Table 1 for an example of the labeled sequences. It should be noted that an intrusion trace contains many normal sequences in addition to the abnormal sequences since the illegal activities only occur in some places within a trace.

We applied RIPPER [Coh95], a rule learning program, to our training data. The following learning tasks were formulated to induce the rule sets for normal and abnormal system call sequences:

- Each record has $n$ positional attributes, $p_{1}, p_{2}, \ldots$, $p_{n}$, one for each of the system calls in a sequence of length $n$; plus a class label, "normal" or "abnormal"

- The training data is composed of normal sequences taken from $80 \%$ of the normal traces, plus the abnormal sequences from 2 traces of the sscp attacks, 1 trace of the syslog-local attack, and 1 trace of the syslog-remote attack

- The testing data includes both normal and abnormal traces not used in the training data.

RIPPER outputs a set of if-then rules for the "minority" classes, and a default "true" rule for the remaining class. The following exemplar RIPPER rules were generated from the system call data:

normal:- $p_{2}=104, p_{7}=112$. [meaning: if $p_{2}$ is 104 (vtimes) and $p_{7}$ is 112 (vtrace) then the sequence is "normal"] 
normal:- $p_{6}=19, p_{7}=105$. [meaning: if $p_{6}$ is 19 (lseek) and $p_{7}$ is 105 (sigvec) then the sequence is "normal"]

...

abnormal:- true. [meaning: if none of the above, the sequence is "abnormal"]

These RIPPER rules can be used to predict whether a sequence is "abnormal" or "normal". But what the intrusion detection system needs to know is whether the trace being analyzed is an intrusion or not. We use the following post-processing scheme to detect whether a given trace is an intrusion based on the RIPPER predictions of its constituent sequences:

1. Use a sliding window of length $2 l+1$, e.g., $7,9,11$, 13 , etc., and a sliding (shift) step of $l$, to scan the predictions made by the RIPPER rules on system call sequences.

2. For each of the (length $2 l+1$ ) regions of RIPPER predictions generated in Step 1, if more than $l$ predictions are "abnormal" then the current region of predictions is an "abnormal" region. (Note that $l$ is an input parameter).

3. If the percentage of abnormal regions is above a threshold value, say $2 \%$, then the trace is an intrusion.

This scheme is an attempt to filter out the spurious prediction errors. The intuition behind this scheme is that when an intrusion actually occurs, the majority of adjacent system call sequences are abnormal; whereas the prediction errors tend to be isolated and sparse. In [FHSL96], the percentage of the mismatched sequences (out of the total number of matches (lookups) performed for the trace) is used to distinguish normal from abnormal. The "mismatched" sequences are the abnormal sequences in our context. Our scheme is different in that we look for abnormal regions that contain more abnormal sequences than the normal ones, and calculate the percentage of abnormal regions (out of the total number of regions). Our scheme is more sensitive to the temporal information, and is less sensitive to noise (errors).

RIPPER only outputs rules for the "minority" class. For example, in our experiments, if the training data has fewer abnormal sequences than the normal ones, the output RIPPER rules can be used to identify abnormal sequences, and the default (everything else) prediction is normal. We conjectured that a set of specific rules for normal sequences can be used as the "identity" of a program, and thus can be used to detect any known and unknown intrusions (anomaly intrusion detection). Whereas having only the rules for abnormal sequences only gives us the capability to identify known intrusions (misuse intrusion detection).

\begin{tabular}{|l|c|c|c|c|c|}
\hline & \% abn. & \multicolumn{4}{|c|}{ \% abn. in experiment } \\
\hline Traces & [FHSL96] & A & B & C & D \\
\hline sscp-1 & 5.2 & 41.9 & 32.2 & 40.0 & 33.1 \\
\hline sscp-2 & 5.2 & 40.4 & 30.4 & 37.6 & 33.3 \\
\hline sscp-3 & 5.2 & 40.4 & 30.4 & 37.6 & 33.3 \\
\hline syslog-r-1 & 5.1 & 30.8 & 21.2 & 30.3 & 21.9 \\
\hline syslog-r-2 & 1.7 & 27.1 & 15.6 & 26.8 & 16.5 \\
\hline syslog-l-1 & 4.0 & 16.7 & 11.1 & 17.0 & 13.0 \\
\hline syslog-1-2 & 5.3 & 19.9 & 15.9 & 19.8 & 15.9 \\
\hline decode-1 & 0.3 & 4.7 & 2.1 & 3.1 & 2.1 \\
\hline decode-2 & 0.3 & 4.4 & 2.0 & 2.5 & 2.2 \\
\hline sm565a & 0.6 & 11.7 & 8.0 & 1.1 & 1.0 \\
\hline sm5x & 2.7 & 17.7 & 6.5 & 5.0 & 3.0 \\
\hline sendmail & 0 & 1.0 & 0.1 & 0.2 & 0.3 \\
\hline
\end{tabular}

Table 2: Comparing Detection of Anomalies. The column [FHSL96] is the percentage of the abnormal sequences of the traces. Columns A, B, C, and D are the percentages of abnormal regions (as measured by the post-processing scheme) of the traces. sendmail is the $20 \%$ normal traces not used in the training data. Traces in bold were included in the training data, the other traces were used as testing data only.

We compare the results of the following experiments that have different distributions of abnormal versus normal sequences in the training data:

Experiment A: $46 \%$ normal and 54\% abnormal, sequence length is 11 ;

Experiment B: $46 \%$ normal and $54 \%$ abnormal, sequence length is 7 ;

Experiment C: $46 \%$ abnormal and $54 \%$ normal, sequence length is 11 ;

Experiment D: $46 \%$ abnormal and 54\% normal, sequence length is 7 .

Table 2 shows the results of using the classifiers from these experiments to analyze the traces. We report here the percentage of abnormal regions (as measured by our post-processing scheme) of each trace, and compare our results with Forrest et al., as reported in [FHSL96]. From Table 2, we can see that in general, intrusion traces generate much larger percentages of abnormal regions than the normal traces. We call these measured percentages the "scores" of the traces. In order to establish a threshold score for identifying intrusion traces, it is desirable that there is a sufficiently large gap between the 
scores of the normal sendmail traces and the low-end scores of the intrusion traces. Comparing experiments that used the same sequence length, we observe that such a gap in $\mathrm{A}, 3.4$, is larger than the gap in C, 0.9; and 1.9 in $\mathrm{B}$ is larger than 0.7 in D. The RIPPER rules from experiments $\mathrm{A}$ and $\mathrm{B}$ describe the patterns of the normal sequences. Here the results show that these rules can be used to identify the intrusion traces, including those not seen in the training data, namely, the decode traces, the sm565a and sm5x traces. This confirms our conjecture that rules for normal patterns can be used for anomaly detection. The RIPPER rules from experiments $\mathrm{C}$ and D specify the patterns of abnormal sequences in the intrusion traces included in the training data. The results indicate that these rules are very capable of detecting the intrusion traces of the "known" types (those seen in the training data), namely, the sscp-3 trace, the syslog-remote- 2 trace and the syslog-local-2 trace. But comparing with the rules from $\mathrm{A}$ and $\mathrm{B}$, the rules in $\mathrm{C}$ and D perform poorly on intrusion traces of "unknown" types. This confirms our conjecture that rules for abnormal patterns are good for misuse intrusion detection, but may not be as effective in detecting future ("unknown") intrusions.

The results from Forrest et al. showed that their method required a very low threshold in order to correctly detect the decode and $s m 565 a$ intrusions. While the results here show that our approach generated much stronger "signals" of anomalies from the intrusion traces, it should be noted that their method used all of the normal traces but not any of the intrusion traces in training.

\subsubsection{Learning to Predict System Calls}

Unlike the experiments in Section 2.1.2 which required abnormal traces in the training data, here we wanted to study how to compute an anomaly detector given just the normal traces. We conducted experiments to learn the (normal) correlation among system calls: the $n$th system calls or the middle system calls in (normal) sequences of length $n$.

The learning tasks were formulated as follows:

- Each record has $n-1$ positional attributes, $p_{1}, p_{2}$, $\ldots, p_{n-1}$, each being a system call; plus a class label, the system call of the $n$th position or the middle position

- The training data is composed of (normal) sequences taken from $80 \%$ of the normal sendmail traces
- The testing data is the traces not included in the training data, namely, the remaining $20 \%$ of the normal sendmail traces and all the intrusion traces.

RIPPER outputs rules in the following form:

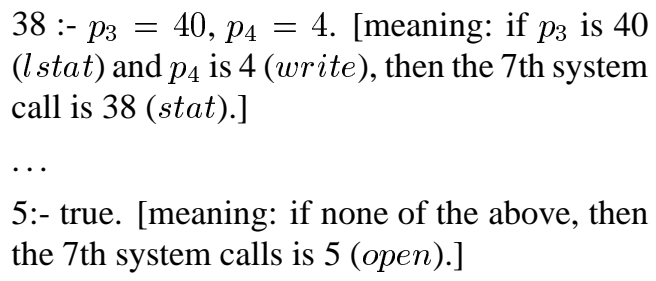

Each of these RIPPER rules has some "confidence" information: the number of matched examples (records that conform to the rule) and the number of unmatched examples (records that are in conflict with the rule) in the training data. For example, the rule for "38 (stat)" covers 12 matched examples and 0 unmatched examples. We measure the confidence value of a rule as the number of matched examples divided by the sum of matched and unmatched examples. These rules can be used to analyze a trace by examining each sequence of the trace. If a violation occurs (the actual system call is not the same as predicted by the rule), the "score" of the trace is incremented by 100 times the confidence of the violated rule. For example, if a sequence in the trace has $p_{3}=40$ and $p_{4}=4$, but $p_{7}=44$ instead of 38 , the total score of the trace is incremented by 100 since the confidence value of this violated rule is 1 . The averaged score (by the total number of sequences) of the trace is then used to decide whether an intrusion has occurred.

Table 3 shows the results of the following experiments:

Experiment A: predict the 11th system call;

Experiment B: predict the middle system call in a sequence of length 7 ;

Experiment C: predict the middle system call in a sequence of length 11 ;

Experiment D: predict the 7th system call.

We can see from Table 3 that the RIPPER rules from experiments A and B are effective because the gap between the score of normal sendmail and the low-end scores of intrusion traces, 3.9 , and 3.3 respectively, are large enough. However, the rules from $\mathrm{C}$ and $\mathrm{D}$ perform poorly. Since $C$ predicts the middle system call of a sequence of length 11 and D predicts the 7th system call, we reason that the training data (the normal traces) has no stable patterns for the 6 th or 7 th position in system call sequences. 


\begin{tabular}{|l|c|c|c|c|}
\hline & \multicolumn{4}{|c|}{ averaged score of violations } \\
\hline Traces & Exp. A & Exp. B & Exp. C & Exp. D \\
\hline sscp-1 & 24.1 & 13.5 & 14.3 & 24.7 \\
\hline sscp-2 & 23.5 & 13.6 & 13.9 & 24.4 \\
\hline sscp-3 & 23.5 & 13.6 & 13.9 & 24.4 \\
\hline syslog-r-1 & 19.3 & 11.5 & 13.9 & 24.0 \\
\hline syslog-r-2 & 15.9 & 8.4 & 10.9 & 23.0 \\
\hline syslog-1-1 & 13.4 & 6.1 & 7.2 & 19.0 \\
\hline syslog-1-2 & 15.2 & 8.0 & 9.0 & 20.2 \\
\hline decode-1 & 9.4 & 3.9 & 2.4 & 11.3 \\
\hline decode-2 & 9.6 & 4.2 & 2.8 & 11.5 \\
\hline sm565a & 14.4 & 8.1 & 9.4 & 20.6 \\
\hline sm5x & 17.2 & 8.2 & 10.1 & 18.0 \\
\hline sendmail & 5.7 & 0.6 & 1.2 & 12.6 \\
\hline
\end{tabular}

Table 3: Detecting Anomalies using Predicted System Calls. Columns A, B, C, and D are the averaged scores of violations of the traces. sendmail is the $20 \%$ normal traces not used in the training data. None of the intrusion traces was used in training.

\subsubsection{Discussion}

Our experiments showed that the normal behavior of a program execution can be established and used to detect its anomalous usage. This confirms the results of other related work in anomaly detection. The weakness of the model in [FHSL96] may be that the recorded (rote learned) normal sequence database may be too specific as it contains $\sim 1,500$ entries. Here we show that a machine learning program, RIPPER, was able to generalize the system call sequence information, from $80 \%$ of the normal sequences, to a set of concise and accurate rules (the rule sets have 200 to 280 rules, and each rule has 2 or 3 attribute tests). We demonstrated that these rules were able to identify unseen intrusion traces as well as normal traces.

We need to search for a more predictive classification model so that the anomaly detector has higher confidence in flagging intrusions. Improvement in accuracy can come from adding more features, rather than just the system calls, into the models of program execution. For example, the directories and the names of the files touched by a program can be used. In [Fra94], it is reported that as the number of features increases from 1 to 3 , the classification error rate of their network intrusion detection system decreases dramatically. Furthermore, the error rate stabilizes after the size of the feature set reaches 4 , the optimal size in their experiments. Many operating systems provide auditing utilities, such as the BSM audit of Solaris, that can be configured to collect abundant information (with many features) of the activities in a host system. From the audit trails, information about a process (program) or a user can then be extracted. The challenge now is to efficiently compute accurate patterns of programs and users from the audit data.

A key assumption in using a learning algorithm for anomaly detection (and to some degree, misuse detection) is that the training data is nearly "complete" with regard to all possible "normal" behavior of a program or user. Otherwise, the learned detection model can not confidently classify or label an unmatched data as "abnormal" since it can just be an unseen "normal" data. For example, the experiments in Section 2.1.3 used 80\% of "normal" system call sequences; whereas the experiments in Section 2.1.2 actually required all "normal" sequences in order to pre-label the "abnormal" sequences to create the training data. During the audit data gathering process, we want to ensure that as much different normal behavior as possible is captured. We first need to have a simple and incremental (continuously learning) summary measure of an audit trail so that we can update this measure as each new audit trail is processed, and can stop the audit process when the measure stabilizes. In Section 3, we propose to use the frequent intraand inter- audit record patterns as the summary measure of an audit trail, and describe the algorithms to compute these patterns.

\subsection{Experiments on tcpdump Data}

There are two approaches for network intrusion detection: one is to analyze the audit data on each host of the network and correlate the evidence from the hosts. The other is to monitor the network traffic directly using a packet capturing program such as tcpdump [JLM89]. In this section, we describe how classifiers can be induced from tcpdump data to distinguish network attacks from normal traffic.

\subsubsection{The tcpdump Data}

We obtained a set of tcpdump data, available via http at "iris.cs.uml.edu:8080/network.html", that is part of an Information Exploration Shootout (see "http://iris.cs.uml.edu:8080"). tcpdump was executed on the gateway that connects the enterprise LAN and the external networks. It captured the headers (not the user data) of the network packets that passed by the network 
interface of the gateway. Network traffic between the enterprise LAN and external networks, as well as the broadcast packets within the LAN were therefore collected. For the purposes of the shootout, filters were used so that tcpdump only collected Internet Transmission Control Protocol (TCP) and Internet User Datagram Protocol (UDP) packets. The data set consists of 3 runs of tcpdump on generated network intrusions ${ }^{1}$ and one tcpdump run on normal network traffic (with no intrusions). The output of each tcpdump run is in a separate file. The traffic volume (number of network connections) of these runs are about the same. Our experiments focused on building an anomaly detection model from the normal dataset.

Since tcpdump output is not intended specifically for security purposes, we had to go through multiple iterations of data pre-processing to extract meaningful features and measures. We studied TCP/IP and its security related problems, for example [Ste84, Pax97, $\mathrm{ABH}^{+}$96, Pax98, Bel89, PV98], for guidelines on the protocols and the important features that characterize a connection.

\subsubsection{Data Pre-processing}

We developed a script to scan each tcpdump data file and extract the "connection" level information about the network traffic. For each TCP connection, the script processes packets between the two ports of the participating hosts, and:

- checks whether 3-way handshake has been properly followed to establish the connection. The following errors are recorded: connection rejected, connection attempted but not established (the initiating host never receives a SYN acknowledgment), and unwanted SYN acknowledgment received (no connection request, a SYN packet, was sent first),

- monitors each data packet and ACK packet, keeps a number of counters in order to calculate these statistics of the connection: resent rate, wrong resent rate, duplicate $\mathrm{ACK}$ rate, hole rate, wrong (data packet) size rate, (data) bytes sent in each direction, percentage of data packet, and percentage of control packet, and

- watches how connection is terminated: normal (both sides properly send and receive FINs), abort (one host sends RST to terminate, and all data pack-

\footnotetext{
${ }^{1}$ Note that, to this date, the organizers of the shootout have not provided us with information, i.e., the times, targets, and actions, of these network intrusions.
}

ets are properly ACKed), half closed (only one host sends FIN), and disconnected.

Since UDP is connectionless (no connection state), we simply treat each packet as a connection.

A connection record, in preparation of data mining, now has the following fields (features): start time, duration, participating hosts, ports, the statistics of the connection (e.g., bytes sent in each direction, resent rate, etc.), flag ("normal" or one of the recorded connection/termination errors), and protocol (TCP or UDP). From the ports, we know whether the connection is to a well-known service, e.g., http (port 80), or a user application.

We call the host that initiates the connection, i.e., the one that sends the first SYN, as the source, and the other as the destination. Depending on the direction from the source to the destination, a connection is in one of the three types: out-going - from the LAN to the external networks; in-coming - from the external networks to the LAN; and inter-LAN - within the LAN. Taking the topologies of the network into consideration is important in network intrusion detection. Intuitively, intrusions (which come from outside) may first exhibit some abnormal patterns (e.g., penetration attempts) in the incoming connections, and subsequently in the inter-LAN (e.g., doing damage to the LAN) and/or the out-going (e.g., stealing/uploading data) connections. Analyzing these types of connections and constructing corresponding detection models separately may improve detection accuracy.

\subsubsection{Experiments and Results}

For each type (direction) of the connections, we formulated the classification experiments as the following:

- Each (connection) record uses the destination service (port) as the class label, and all the other connection features as attributes;

- The training data is $80 \%$ of the connections from the normal tcpdump data file, while the test data includes the remaining $20 \%$ from the normal $t c p$ dump data file, and all the connections from the 3 tcpdump data files marked as having embedded attacks;

- 5-fold cross validation evaluation is reported here. The process (training and testing) is repeated 5 times, each time using a different $80 \%$ of the normal data as the training data (and accordingly the 


\begin{tabular}{|l|c|c|c|}
\hline & \multicolumn{3}{|c|}{$\%$ misclassification (by traffic type) } \\
\hline Data & out-going & in-coming & inter-LAN \\
\hline normal & $3.91 \%$ & $4.68 \%$ & $4 \%$ \\
\hline intrusion1 & $3.81 \%$ & $6.76 \%$ & $22.65 \%$ \\
\hline intrusion2 & $4.76 \%$ & $7.47 \%$ & $8.7 \%$ \\
\hline intrusion3 & $3.71 \%$ & $13.7 \%$ & $7.86 \%$ \\
\hline
\end{tabular}

Table 4: Misclassification Rate on Normal and Intrusion Data. Separate classifiers were trained and tested on connection data of each traffic type. "normal" is the $20 \%$ data set aside from the training data. No intrusion data was used for training.

different remaining $20 \%$ of the normal data as part of the test data), and the averaged accuracy of the classifiers from the 5 runs is reported.

We again applied RIPPER to the connection data. The resulting classifier characterizes the normal patterns of each service in terms of the connection features. When using the classifier on the testing data, the percentage of misclassifications on each tcpdump data set is reported. Here a misclassification is the situation where the the classifier predicts a destination service (according to the connection features) that is different from the actual. This misclassification rate should be very low for normal connection data and high for intrusion data. The intuition behind this classification model is straightforward: when intrusions take place, the features (characteristics) of connections to certain services, for example, $f t p$, are different from the normal traffic patterns (of the same service).

The results from the first round of experiments, as shown in Table 4, were not very good: the differences in the misclassification rates of the normal and intrusion data were small, except for the inter-LAN traffic of some intrusions.

We then redesigned our set of features by adding some continuous and intensity measures into each connection record:

- Examining all connections in the past $n$ seconds, and counting the number of: connection establishment errors (e.g., "connection rejected"), all other types of errors (e.g., "disconnected"), connections to designated system services (e.g., ftp), connections to user applications, and connections to the same service as the current connection;

- Calculate for the past $n$ seconds, the per-connection average duration and data bytes (on both directions) of all connections, and the same averages of con-

\begin{tabular}{|l|c|c|c|}
\hline & \multicolumn{3}{|c|}{$\%$ misclassification (by traffic type) } \\
\hline Data & out-going & in-coming & inter-LAN \\
\hline normal & $0.88 \%$ & $0.31 \%$ & $1.43 \%$ \\
\hline intrusion1 & $2.54 \%$ & $27.37 \%$ & $20.48 \%$ \\
\hline intrusion2 & $3.04 \%$ & $27.42 \%$ & $5.63 \%$ \\
\hline intrusion3 & $2.32 \%$ & $42.20 \%$ & $6.80 \%$ \\
\hline
\end{tabular}

Table 5: Using Temporal-Statistical Measures to Improve Classification Accuracy. Here the time interval is 30 seconds.

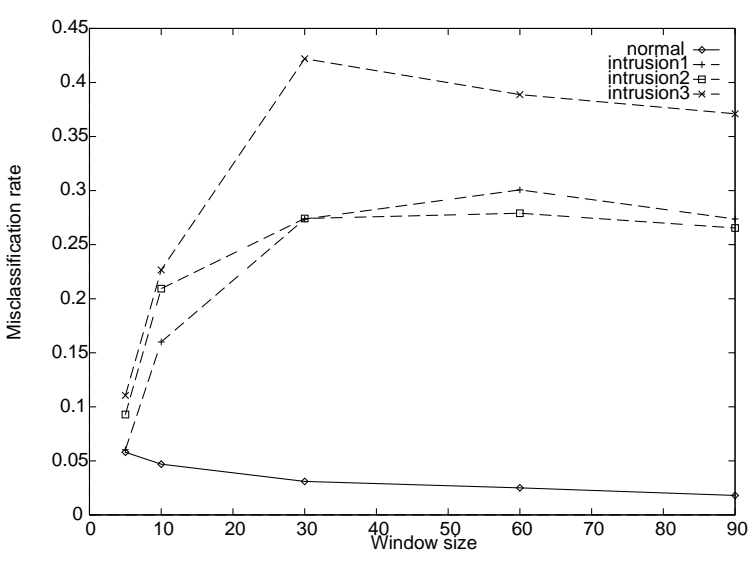

Figure 1: Effects of Window Sizes on Misclassification Rates

nections to the same service.

These additional temporal-statistical features provide additional information of the network activity from a continuous perspective, and provide more insight into anomalies. For example, a low rate of error due to innocent attempts and network glitches in a short time span is expected, but an excess beyond the (averaged) norm indicates anomalous activity. Table 5 shows the improvement of adding these features. Here, using a time interval of 30 seconds (i.e., $n=30 s$ ), we see that the misclassification rates on the intrusion data are much higher than the normal data, especially for the in-coming traffic. The RIPPER rule set (the classifier) has just 9 rules and 25 conditions. For example, one rule says "if the average number of bytes from source to destination (of the connections to the same service) is 0 , and the percentage of control packets in the current connection is $100 \%$, then the service is auth".

To understand the effects of the time intervals on the misclassification rates, we ran the experiments using various time intervals: $5 \mathrm{~s}, 10 \mathrm{~s}, 30 \mathrm{~s}, 60 \mathrm{~s}$, and $90 \mathrm{~s}$. The effects on the out-going and inter-LAN traffic were very small. However, as Figure 1 shows, for the in-coming 
traffic, the misclassification rates on the intrusion data increase dramatically as the time interval goes from $5 \mathrm{~s}$ to 30 s, then stabilizes or tapers off afterwards.

\subsubsection{Discussion}

We learned some important lessons from the experiments on the tcpdump data. First, when the collected data is not designed specifically for security purposes or can not be used directly to build a detection model, a considerable amount of (iterative) data pre-processing is required. This process fundamentally requires a lot of domain knowledge, and may not be easily automated. Second, in general, adding temporal-statistical features can improve the accuracy of the classification model.

There are also much needed improvements to our current approach: First, deciding upon the right set of features is difficult and time consuming. For example, many trials were attempted before we came up with the current set of features and time intervals. We need useful tools that can provide insight into the patterns that may be exhibited in the data. Second, we should provide tools that can help administrative staff understand the nature of the anomalies.

\subsection{Combining Multiple Classifiers}

The classifiers described in this section each models a single aspect of the system behavior. They are what we call the base (single level) classifiers. Combining evidence from multiple base classifiers that each models different aspect of the target system is likely to improve the effectiveness in detecting intrusions. For example, in addition to the classifier for network traffic (using $t c p$ dump data), we can include the classifiers on the commands issued during the (connection) sessions of wellknown services, e.g. ftp, telnet etc. The combined evidence of anomalous traffic patterns and session behavior leads to a more accurate assertion that the network is under attack. A priority in our research plan is to study and experiment with (inductively learned) classification models that combine evidence from multiple (base) detection models. The general approach in learning such a meta-detection model can be summarized as follows:

- Build base classifiers that each models different aspect of the target system;

- Formulate the meta learning task: each record in the training data is a collection of the evidence (generated at the same time period) from the base classifiers; each attribute value in a record is 1 or 0 , the prediction (evidence) from a base classifier that the modeled behavior is "normal" or "abnormal" (i.e., it fits the model or not).

- Apply a learning algorithm to produce the meta classifier.

The meta detection model is actually a hierarchy of detection models. At the bottom, the base classifiers take audit data as input and output evidence to the meta classifier, which in turn outputs the final assertion.

Our research activities in JAM [SPT $\left.{ }^{+} 97\right]$, which focus on the accuracy and efficiency of meta classifiers, will contribute significantly to our effort in building meta detection models.

\section{Mining Patterns from Audit Data}

In order to construct an accurate (effective) base classifier, we need to gather a sufficient amount of training data and identify a set of meaningful features. Both of these tasks require insight into the nature of the audit data, and can be very difficult without proper tools and guidelines. In this section we describe some algorithms that can address these needs. Here we use the term "audit data" to refer to general data streams that have been properly processed for detection purposes. An example of such data streams is the connection record data extracted from the raw tcpdump output.

\subsection{Association Rules}

The goal of mining association rules is to derive multifeature (attribute) correlations from a database table. A simple yet interesting commercial application of the association rules algorithm is to determine what items are often purchased together by customers, and use that information to arrange store layout. Formally, given a set of records, where each record is a set of items, an association rule is an expression $X \Rightarrow$ $Y$, confidence, support [SA95]. $X$ and $Y$ are subsets of the items in a record, support is the percentage of records that contain $X+Y$, whereas confidence is $\frac{\text { support }(X+Y)}{\text { support }(X)}$. For example, an association rule from the shell command history file (which is a stream of com- 
mands and their arguments) of a user is

$$
\text { trn } \Rightarrow \text { rec.humor } ;[0.3,0.1],
$$

which indicates that $30 \%$ of the time when the user invokes trn, he or she is reading the news in rec.humor, and reading this newsgroup accounts for $10 \%$ of the activities recorded in his or her command history file. Here 0.3 is the confidence and 0.1 is the support.

The motivation for applying the association rules algorithm to audit data are:

- Audit data can be formatted into a database table where each row is an audit record and each column is a field (system feature) of the audit records;

- There is evidence that program executions and user activities exhibit frequent correlations among system features. For example, one of the reasons that "program policies", which codify the access rights of privileged programs, are concise and capable to detect known attacks [KFL94] is that the intended behavior of a program, e.g., read and write files from certain directories with specific permissions, is very consistent. These consistent behaviors can be captured in association rules;

- We can continuously merge the rules from a new run to the aggregate rule set (of all previous runs).

Our implementation follows the general association rules algorithm, as described in [Sri96].

\subsection{Frequent Episodes}

While the association rules algorithm seeks to find intraaudit record patterns, the frequent episodes algorithm, as described in [MTV95], can be used to discover inter- audit record patterns. A frequent episode is a set of events that occur frequently within a time window (of a specified length). The events must occur (together) in at least a specified minimum frequency, min_fr, sliding time window. Events in a serial episode must occur in partial order in time; whereas for a parallel episode there is no such constraint. For $X$ and $Y$ where $X+Y$ is a frequent episode, $X \Rightarrow Y$ with confidence $=\frac{\text { frequency }(X+Y)}{\text { frequency }(X)}$ and support $=$ frequency $(X+Y)$ is called a frequent episode rule. An example frequent serial episode rule from the log file of a department's Web site is

$$
\text { home, research } \Rightarrow \text { theory } ;[0.2,0.05],[30 s]
$$

which indicates that when the home page and the research guide are visited (in that order), in $20 \%$ of the cases the theory group's page is visited subsequently within the same 30s time window, and this sequence of visits occurs $5 \%$ of the total (the 30 s) time windows in the $\log$ file (that is, approximately $5 \%$ of all the records).

We seek to apply the frequent episodes algorithm to analyze audit trails since there is evidence that the sequence information in program executions and user commands can be used to build profiles for anomaly detection [FHSL96, LB97]. Our implementation followed the description in [MTV95].

\subsection{Using the Discovered Patterns}

The association rules and frequent episodes can be used to guide the audit process. We run a program many times and under different settings. For each new run, we compute its rule set (that consists of both the association rules and the frequent episodes) from the audit trail, and update the (existing) aggregate rule sets using the following merge process:

- For each rule in the new rule set: find a match in the aggregate rule set. A match is defined as the exact matches on both the LHS and RHS of the rules, plus $\varepsilon$ matches (using ranges), on the support (or frequency) and con fidence values

- If a match is found, increment the match_count of the matched rule in the aggregate rule set. Otherwise, add the new rule and initialize its match_count to be 1 .

When the rule set stabilizes (there are no new rules added), we can stop the data gathering process since we have produced a near complete set of audit data for the normal runs. We then prune the rule set by eliminating the rules with low match_count, according to a userdefined threshold on the ratio of match_count over the total number of audit trails. The system builders can then use the correlation information in this final profile rule set to select a subset of the relevant features for the classification tasks. We plan to build a support environment to integrate the process of user selection of features, computing a classifier (according to the feature set), and presenting the performance of the classifier. Such a support system can speed up the iterative feature selection process, and help ensure the accuracy of a detection model.

We believe that the discovered patterns from (the extensively gathered) audit data can be used directly for anomaly detection. We compute a set of association 


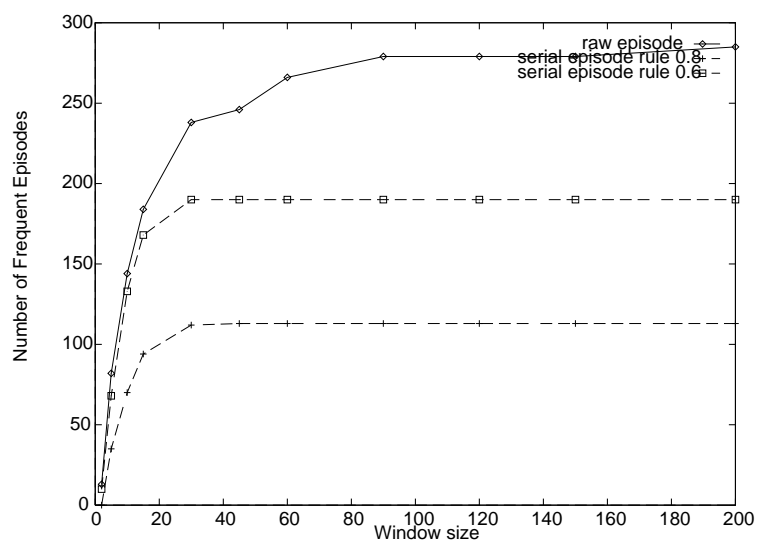

Figure 2: Effects of Window Sizes on the Number of Frequent Episodes.

rules and frequent episodes from a new audit trail, and compare it with the established profile rule set. Scoring functions can be used to evaluate the deviation scores for: missing rules with high support, violation (same antecedent but different consequent) of rules with high support and con fidence, new (unseen) rules, and significant changes in support of rules.

\subsection{1 tcpdump Data Revisited}

We ran some preliminary experiments using our association rules and frequent episodes programs on the $t c p$ dump data that was used in the experiments described in Section 2.2.

We wanted to study how the frequent episodes algorithm can help us determine the time window used in gathering temporal-statistical features. We ran the algorithm on the "normal" in-coming connection records (without the temporal-statistical features). We set the program to produce two types of output: raw serial and parallel episodes (no rules were generated) and serial episode rules. For raw episodes, we used min $_{-} f r=0.3$. And for serial episode rules, we used $\min _{-} f r=0.1$ and min_con $f=0.6$ and 0.8 . We used different time window sizes (win): 2s, 5s, 10s, 15s, 30s, 45s, 60s, 90s, $120 \mathrm{~s}, 150 \mathrm{~s}$, and 200s; and recorded the number of frequent episodes generated on each win. In Figure 2 we see that the number of frequent episodes ( $r a w$ episodes or serial episode rules) increases sharply as win goes from 2 s to $30 \mathrm{~s}$, it then gradually stabilizes (note that by the nature of the frequent episodes algorithm, the number of episodes can only increase as win increases). This phenomenon coincides with the trends in Figure 1. Note that here we made the particular choice of the parame- ters (i.e., min_fr, min_conf) only for the purpose of controlling the maximum size of the episode rule set. Different settings exhibited the same phenomenon. We conjecture (and will verify with further experiments on other data sets) that we can use this technique to analyze data streams and automatically discover the most important temporal measure: the time window size, i.e., the period of time within which to measure appropriate statistical features to maximize classifier accuracy. Intuitively, the first requirement of a time window size is that its set of sequence patterns is stable, that is, sufficient patterns are captured and noise is small.

We also ran both the association rules and frequent episodes programs on all the in-coming connection data, and compared the rule sets from the normal data with the intrusion data. The purpose of this experiment was to determine how these programs can provide insight into the (possible) patterns of intrusions. The frequent episodes generated were serial episode rules with win $=30 \mathrm{~s}$, min_f $_{-}=0.1$ and min_con $f=0.8$. The associations rules were generated using min_support $=0.3$ and min_confidence $=0.9$. We manually examined and compared the rule sets to look for "unique" patterns that exist in the intrusion data (but not in the normal data). Here are some results:

intrusion1: the unique serial rules are related to "ftpdata as the source application”, for example,

$$
\begin{aligned}
& s r c \_s r v=\text { "ftp-data", src_srv = "user- } \\
& \text { apps" } \Longrightarrow s r c \_s r v=\text { "ftp-data"; }[0.96, \\
& 0.11],[30 \mathrm{~s}]
\end{aligned}
$$

This rule means: when a connection with a user application as the source service follows a connection with ftp-data, $96 \%$ of the cases, a connection with ftp-data follows and falls into the same time window (30s); and this patterns occur $11 \%$ of the time. The unique association rules are related to "destination service is a user application", for example,

$$
\begin{aligned}
& \text { dst_srv }=\text { "user-apps" } \Longrightarrow \text { duration }=0, \\
& \text { dst_to_src_bytes }=0 ;[0.9,0.33]
\end{aligned}
$$

This rule means: when the destination service of a connection is a user application, $90 \%$ of the cases, the duration and the number of data bytes from the destination to the source are both 0 ; and this pattern occurs $33 \%$ of the time.

intrusion2: the results are nearly identical to intrusion 1 in terms of the unique serial rules and association rules.

intrusion3: the unique serial rules are related to "auth as the destination service", for example, 
dst_srv = "auth" $\Longrightarrow$ flag = "un-

wanted_syn_ack”; [0.82, 0.1], [30s]

and

$$
\begin{aligned}
& d s t \_s r v=\text { "auth" } \Longrightarrow d s t \_s r v=\text { "user- } \\
& \text { apps", dst_srv = "auth"; [0.82, } 0.1] \text {, } \\
& \text { [30s] }
\end{aligned}
$$

There are a significant number of unique association rules in regard to "smtp is the source application". Many of these rules suggest connection error of smtp, for example,

$$
\begin{aligned}
& \text { src_srv }=\text { "smtp" } \Longrightarrow \text { duration }=0, \text { flag } \\
& =\text { "unwanted_syn_ack", dst_srv }=\text { "user- } \\
& \text { apps"; }[1.0,0.38]
\end{aligned}
$$

These rules may provide hints about the intrusions. For example, the unique (not normal) serial episodes in intrusion 1 and intrusion 2 reveal that there are a large number of $f t p$ data transfer activities; whereas the unique serial episodes in intrusion 3 suggest that a large number of connections to the auth service were attempted.

\section{Architecture Support}

The biggest challenge of using data mining approaches in intrusion detection is that it requires a large amount of audit data in order to compute the profile rule sets. And the fact that we may need to compute a detection model for each resource in a target system makes the data mining task daunting. Moreover, this learning (mining) process is an integral and continuous part of an intrusion detection system because the rule sets used by the detection module may not be static over a long period of time. For example, as a new version of a system software arrives, we need to update the "normal" profile rules. Given that data mining is an expensive process (in time and storage), and real-time detection needs to be lightweight to be practical, we can't afford to have a monolithic intrusion detection system.

We propose a system architecture, as shown in Figure 3, that includes two kinds of intelligent agents: the learning agents and the detection agents. A learning agent, which may reside in a server machine for its computing power, is responsible for computing and maintaining the rule sets for programs and users. It produces both the base detection models and the meta detection models. The task of a learning agent, to compute accurate models from very large amount of audit data, is an example of the "scale-up" problem in machine learning. We expect that our research in agent-based metalearning systems [SPT ${ }^{+}$97] will contribute significantly to the implementation of the learning agents. Briefly, we are studying how to partition and dispatch data to a host of machines to compute classifiers in parallel, and re-import the remotely learned classifiers and combine an accurate (final) meta-classifier, a hierarchy of classifiers [CS93].

A detection agent is generic and extensible. It is equipped with a (learned and periodically updated) rule set (i.e., a classifier) from the remote learning agent. Its detection engine "executes" the classifier on the input audit data, and outputs evidence of intrusions. The main difference between a base detection agent and the meta detection agent is: the former uses preprocessed audit data as input while the later uses the evidence from all the base detection agents. The base detection agents and the meta detection agent need not be running on the same host. For example, in a network environment, a meta agent can combine reports from (base) detection agents running on each host, and make the final assertion on the state of the network.

The main advantages of such a system architecture are:

- It is easy to construct an intrusion detection system as a compositional hierarchy of generic detection agents.

- The detection agents are lightweight since they can function independently from the heavyweight learning agents, in time and locale, so long as it is already equipped with the rule sets.

- A detection agent can report new instances of intrusions by transmitting the audit records to the learning agent, which can in turn compute an updated classifier to detect such intrusions, and dispatch them to all detection agents. Interestingly, the capability to derive and disseminate anti-virus codes faster than the virus can spread is also considered a key requirement for anti-virus systems [KSSW97].

\section{Conclusion and Future Work}

In this paper we proposed a systemic framework that employs data mining techniques for intrusion detection. This framework consists of classification, association rules, and frequence episodes programs, that can be used 


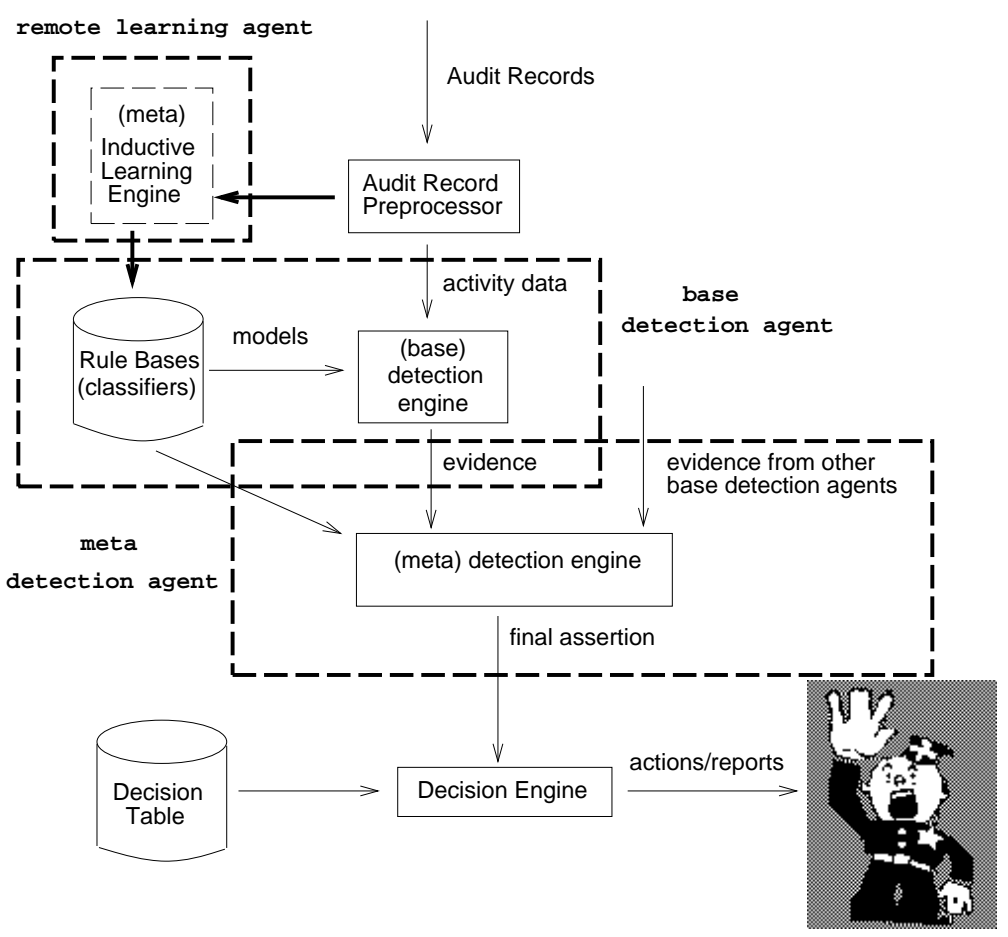

Figure 3: An Architecture for Agent-Based Intrusion Detection System

to (automatically) construct detection models. The experiments on sendmail system call data and network tcpdump data demonstrated the effectiveness of classification models in detecting anomalies. The accuracy of the detection models depends on sufficient training data and the right feature set. We suggested that the association rules and frequent episodes algorithms can be used to compute the consistent patterns from audit data. These frequent patterns form an abstract summary of an audit trail, and therefore can be used to: guide the audit data gathering process; provide help for feature selection; and discover patterns of intrusions. Preliminary experiments of using these algorithms on the tcpdump data showed promising results.

We are in the initial stages of our research, much remains to be done including the following tasks:

- Implement a support environment for system builders to iteratively drive the integrated process of pattern discovering, system feature selection, and construction and evaluation of detection models;

- Investigate the methods and benefits of combining multiple simple detection models. We need to use multiple audit data streams for experiments;

- Implement a prototype agent-based intrusion detection system. JAM [SPT ${ }^{+}$97] already provides a base infrastructure;
- Evaluate our approach using extensive audit data sets, some of which is presently under construction at Rome Labs.

\section{Acknowledgments}

We are very grateful to Stephanie Forrest and Steven A. Hofmeyr, both of University of New Mexico, for providing us with the system call data and explaining the details of their experiments. We also wish to thank Philip K. Chan of Florida Institute of Technology and David Wei Fan of Columbia University for their helpful advice.

\section{References}

[ABH ${ }^{+96]}$ D. Atkins, P. Buis, C. Hare, R. Kelley, C. Nachenberg, A. B. Nelson, P. Phillips, T. Ritchey, and W. Steen. Internet Security Professional Reference. New Riders Publishing, 1996.

[Bel89] S. M. Bellovin. Security problems in the tcp/ip protocol suite. Computer Communication Review, 19(2):32-48, April 1989. 
[Coh95] W. W. Cohen. Fast effective rule induction. In Machine Learning: the 12th International Conference, Lake Taho, CA, 1995. Morgan Kaufmann.

[CS93] P. K. Chan and S. J. Stolfo. Toward parallel and distributed learning by meta-learning. In AAAI Workshop in Knowledge Discovery in Databases, pages 227-240, 1993.

[FHSL96] S. Forrest, S. A. Hofmeyr, A. Somayaji, and T. A. Longstaff. A sense of self for unix processes. In Proceedings of the 1996 IEEE Symposium on Security and Privacy, pages 120-128, Los Alamitos, CA, 1996. IEEE Computer Society Press.

[FPSS96] U. Fayyad, G. Piatetsky-Shapiro, and P. Smyth. The KDD process of extracting useful knowledge from volumes of data. Communications of the ACM, 39(11):2734, November 1996.

[Fra94] J. Frank. Artificial intelligence and intrusion detection: Current and future directions. In Proceedings of the 17th $\mathrm{Na}$ tional Computer Security Conference, October 1994.

[HLMS90] R. Heady, G. Luger, A. Maccabe, and M. Servilla. The architecture of a network level intrusion detection system. Technical report, Computer Science Department, University of New Mexico, August 1990.

[IKP95] K. Ilgun, R. A. Kemmerer, and P. A. Porras. State transition analysis: A rule-based intrusion detection approach. IEEE Transactions on Software Engineering, 21(3):181199, March 1995.

[JLM89] V. Jacobson, C. Leres, and S. McCanne. tcpdump. available via anonymous ftp to ftp.ee.lbl.gov, June 1989.

[KFL94] C. Ko, G. Fink, and K. Levitt. Automated detection of vulnerabilities in privileged programs by execution monitoring. In Proceedings of the 10th Annual Computer Security Applications Conference, pages 134144, December 1994.

[KS95] S. Kumar and E. H. Spafford. A software architecture to support misuse intrusion detection. In Proceedings of the 18th National Information Security Conference, pages 194204, 1995.
[KSSW97] J. O. Kephart, G. B. Sorkin, M. Swimmer, and S. R. White. Blueprint for a computer immune system. Technical report, IBM T. J. Watson Research Center, Yorktown Heights, New York, 1997.

[LB97] T. Lane and C. E. Brodley. Sequence matching and learning in anomaly detection for computer security. In AAAI Workshop: AI Approaches to Fraud Detection and Risk Management, pages 43-49. AAAI Press, July 1997.

[LSC97] W. Lee, S. J. Stolfo, and P. K. Chan. Learning patterns from unix process execution traces for intrusion detection. In $A A A I$ Workshop: AI Approaches to Fraud Detection and Risk Management, pages 50-56. AAAI Press, July 1997.

[LTG ${ }^{+92]}$ T. Lunt, A. Tamaru, F. Gilham, R. Jagannathan, P. Neumann, H. Javitz, A. Valdes, and T. Garvey. A real-time intrusion detection expert system (IDES) - final technical report. Technical report, Computer Science Laboratory, SRI International, Menlo Park, California, February 1992.

[MTV95] H. Mannila, H. Toivonen, and A. I. Verkamo. Discovering frequent episodes in sequences. In Proceedings of the 1st International Conference on Knowledge Discovery in Databases and Data Mining, Montreal, Canada, August 1995.

[Pax97] Vern Paxon. End-to-end internet packet dynamics. In Proceedings of SIGCOMM '97, September 1997.

[Pax98] Vern Paxon. Bro: A system for detecting network intruders in real-time. In Proceedings of the 7th USENIX Security Symposium, San Antonio, TX, 1998.

[PV98] Phillip A. Porras and Alfonso Valdes. Live traffic analysis of tcp/ip gateways. In Proceedings of the Internet Society Symposium on Network and Distributed System Security, March 1998.

[SA95] R. Srikant and R. Agrawal. Mining generalized association rules. In Proceedings of the 21st VLDB Conference, Zurich, Switzerland, 1995.

[SPT ${ }^{+97]}$ S. J. Stolfo, A. L. Prodromidis, S. Tselepis, W. Lee, D. W. Fan, and P. K. Chan. 
Jam: Java agents for meta-learning over distributed databases. In Proceedings of the 3 rd International Conference on Knowledge Discovery and Data Mining, pages 74-81, Newport Beach, CA, August 1997. AAAI Press.

[Sri96] R. Srikant. Fast Algorithms for Mining Association Rules and Sequential Patterns. $\mathrm{PhD}$ thesis, University of Wisconsin - Madison, 1996.

[Ste84] W. R. Stevens. TCP/IP Illustrated, volume 1. Addison-Wesley Publishing Company, 1984. 